\title{
Immunoglobulin-E Mediated Chlorhexidine Hypersensitivity among Healthcare Workers
}

\section{Alam SMJ* and Thayalan S \\ St George's University Hospital, London, UK}

*Corresponding author: Syed Muhammed Junaid Alam, P0 Box 20655, Building 3304, Dhahran 31311, Saudi Arabia, Tel: +966 559252597; Email: junaid@doctors.org.uk

\section{Research Article \\ Volume 5 Issue 5}

Received Date: October 07, 2021

Published Date: October 28, 2021

DOI: $10.23880 /$ hhij-16000251

\section{Abstract}

Objective: With a wide spectrum antimicrobial activity that is not affected by body fluids and blood, chlorhexidine is a particularly useful disinfectant widely used in healthcare settings. Given the importance of disinfectant usage by healthcare workers to prevent nosocomial infections, particularly during the COVID-19 pandemic, this study aims to evaluate the prevalence of immunoglobulin E (IgE)-mediated sensitivity in healthcare workers.

Methodology: A cross-sectional study was conducted through a questionnaire and immunoassay for chlorhexidinespecific IgE in healthcare workers in a large teaching hospital in London, UK. The prevalence of IgE-mediated chlorhexidine hypersensitivity was determined along with potential determinants.

Results: With a 77.7\% response rate, the study showed that 4 out of 233 participants were positive for chlorhexidine-specific IgE, with an overall prevalence of $1.72 \%$ (95\% Confidence Interval: $0.05 \%$ to $3.39 \%$ ). All the positive cases belonged to occupationally exposed healthcare workers. In this group, the prevalence rate was $2.82 \%$ (95\% Confidence Interval: $0.1 \%$ to $5.54 \%)$.

Conclusion: Chlorhexidine is a potential allergen and an under-reported cause of anaphylaxis in patient-care and occupational settings. The results suggest that chlorhexidine has a relatively safe profile for healthcare workers in occupational settings, but a higher prevalence of chlorhexidine hypersensitivity cannot be ruled out. Sensitized healthcare workers must be advised to avoid further exposure to prevent potentially serious IgE-mediated allergic symptoms. Further studies are recommended to determine if any change in disinfection guidelines and protocols is warranted.

Keywords: Chlorhexidine; Immediate hypersensitivity; Occupational illness; Health personnel; COVID-19

\section{Introduction}

Chlorhexidine is a broad-spectrum antimicrobial biguanide which, since 1954, has been used in healthcare and dental practice for topical application or oral rinse to achieve antisepsis and treat certain conditions such as gingivitis [1-4]. The antibacterial activity of chlorhexidine is concentrationdependent, the main target being the bacterial cytoplasmic membrane $[5,6]$. At lower concentrations, it is bacteriostatic (e.g., at $1 \mu \mathrm{g} / \mathrm{ml}$ to $2.5 \mu \mathrm{g} / \mathrm{ml}$ ) [7-9]. At concentrations of $\geq 20$ $\mu \mathrm{g} / \mathrm{ml}$, chlorhexidine has a bactericidal activity [10]. Because of its relatively high antimicrobial activity, which is not affected by body fluids such as blood [11], chlorhexidine is frequently used by healthcare workers in operating theatres and emergency departments. 
In addition, chlorhexidine has fungicidal and fungistatic activity. Furthermore, it is effective against enveloped viruses such as SARS-CoV-2, human immunodeficiency virus, herpes virus, respiratory syncytial virus, influenza virus, and cytomegalovirus. In a recent study published in 2021 [12], chlorhexidine used as oral rinse was significantly effective in eliminating oropharyngeal SARS-CoV-2, responsible for the COVID-19 pandemic. It suggests that chlorhexidine has the potential usefulness for reducing the spread of the COVID-19 pandemic in healthcare settings.

Being frequent and regular users of chlorhexidine, healthcare workers must be aware of its adverse reactions, including hypersensitivity reactions. Chlorhexidine can cause type I (IgE-mediated) as well as type IV (cell-mediated) hypersensitivity. Skin reactions include contact and allergic contact dermatitis [13-19], contact urticarial [20], and photosensitive dermatitis [21].

Taking into account its widespread use in healthcare settings, serious allergic reactions to chlorhexidine are relatively uncommon [22] but can be a serious problem in patients undergoing anesthesia and surgical procedures [23]. The clinical picture ranges from urticaria to full-blown anaphylaxis [24]. Acute hypersensitivity to chlorhexidine has been reported using chlorhexidine as a skin antiseptic for surgery [23,25], insertion of epidural catheters [26] or urological catheterization [27,28]; lubricant gel [27]; chlorhexidine-coated central venous catheters [29-32]; over-the-counter antiseptics; and chlorhexidine antiseptic for mucous membranes.

Although there are several reports of an acute hypersensitivity reaction among patients, relatively few studies have estimated the prevalence of chlorhexidine hypersensitivity in healthcare workers in occupational settings. Sensitization to chlorhexidine in the workplace may occur after mucosal or skin application or inhalation. As in non-occupational settings, both type I and IV chlorhexidine hypersensitivities can occur in occupational settings. Most studies on occupational exposure to chlorhexidine have dealt with the effects of the irritant or allergic contact dermatitis (type IV hypersensitivity) [33].

Type IV (cell-mediated) hypersensitivity reactions are more often, but IgE-mediated type I hypersensitivity is more serious and can lead to severe and life-threatening anaphylactic reactions. Only a few published studies have attempted to estimate the prevalence of IgE-mediated chlorhexidine hypersensitivity in healthcare workers [3436]. A case study described two nurses who developed asthma after exposure to a surface disinfectant spray containing chlorhexidine and alcohol [36]. Similarly, in 2018, a case report was published in Italy that described type 1 chlorhexidine hypersensitivity in a dentist [37]. In a survey, detergents containing chlorhexidine were reported to cause skin damage among nurses. In a questionnaire-based survey, 89 out of 307 physicians reported occupational allergies, and $23(7.5 \%)$ implicated chlorhexidine as an allergen, which was only second to surgical gloves as the most common cause of self-reported allergic symptoms [38].

Garvey, et al. failed to identify a single positive case of occupational allergy to chlorhexidine in 104 Danish healthcare workers using a skin prick test (SPT) for type I IgE-mediated hypersensitivity and patch tests for type IV hypersensitivity [34]. In that study, the Danish healthcare workers used products with lower concentrations $(0.5 \%-1.0 \%)$ of chlorhexidine. By contrast, healthcare workers elsewhere, such as the UK, are exposed to up to $4 \%$ chlorhexidine concentrations. Nagendran, et al. demonstrated that $4(28 \%)$ of the 14 symptomatic healthcare workers in the UK were positive for chlorhexidine-specific IgE [39]. Although the sample size was small, the study raised questions about chlorhexidine hypersensitivity and potential occupational health implications for healthcare workers who use it frequently.

With increased emphasis on the prevention of healthcareassociated infections and control measures for the COVD-19 pandemic, healthcare workers are exposed to chlorhexidine now more than ever. Therefore, a study to determine the prevalence of IgE-mediated chlorhexidine sensitization among healthcare workers is justified to assess the safety of chlorhexidine in occupational settings and whether changes in current guidelines for disinfectant usage are warranted to avoid or minimize its potential health impact.

\section{Methods}

This descriptive, cross-sectional study used a quantitative analysis (immunoassay for chlorhexidinespecific IgE levels) and a self-administered questionnaire. The sample population was a cohort of healthcare workers from a tertiary care teaching hospital in London, United Kingdom.

\section{Inclusion Criteria}

Adult healthcare workers attending the Occupational Health Department of large teaching hospital in London (United Kingdom) were included, irrespective of their health status and reason for attendance.

\section{Healthcare Worker: Definition}

In this study, the definition of a healthcare worker was derived from the guidance notes of the Association of National Health Occupational Physicians-UK, which divides 
healthcare workers into three categories as follows:

- Clinical and other staff, including those in primary care, who have regular clinical contact with patients: physicians, dentists, and nurses; paramedical professionals such as occupational therapists, physiotherapists, radiographers, ambulance workers, and porters; and students in these disciplines.

- Laboratory and other staff who has direct contact with potentially infectious clinical specimens and may be exposed to pathogens in the laboratory.

- Nonclinical ancillary staff that may have social contact with patients but usually not of a prolonged or close nature.

\section{Sample Size Calculation}

Two previous studies [34,39] demonstrated $0 \%$ and $28 \%$ positive results for IgE-mediated hypersensitivity. Hence, the expected prevalence or proportion (P) for this study was set at $20 \%$. The sample size was calculated using the following simple formula [40]:

$$
n=\frac{Z^{2} P(1-P)}{d^{2}}
$$

$\mathrm{n}=\left(\mathrm{Z}^{\wedge} 2 \mathrm{P}(1-\mathrm{P})\right) / \mathrm{d}^{\wedge} 2$

$\mathrm{n}=$ sample size

$\mathrm{Z}=\mathrm{Z}$ statistic for a level of confidence (for $95 \%$ confidence interval [CI], 1.96)

$\mathrm{P}=$ expected prevalence or proportion (in proportion of one; $20 \%, \mathrm{p}=0.2$ )

$d=$ precision or margin of error (in proportion of one, $5 \% d$ $=0.05$ )

$$
n=\frac{1.96^{2} \times 0.2(1-0.2)}{0.05^{2}} \text { or } \frac{0.77(0.8)}{0.0025}=246
$$

$\mathrm{n}=\left([1.96) \rrbracket^{\wedge} 2 \times 0.2(1-0.2)\right) /[0.05]^{\wedge} 2$ or $(0.77(0.8)) / 0.0025$ $=246$

A sample size of approximately 250 participants was set as the target with an expected prevalence of 20\% (95\% CI: $15.5 \%-25.4 \%$ ). Increasing the sample size to 400 does not lead to a significant narrowing of the interval.

\section{Assessment Tools}

Questionnaire: The author designed a questionnaire after consultation with an immunologist, a library statistician, and his supervisor. The questionnaire was modified and finalized after it was piloted on 15 healthcare workers.

Chlorhexidine-Specific IgE immunoassay: The study used an in vitro immunoassay to detect chlorhexidine-specific IgE levels using 5-8 mL of venous blood. The serum level of chlorhexidine-specific IgE antibodies was measured using the sandwich immunoassay (solid-phase ImmunoCAP ${ }^{\mathrm{TM}}$; Allergen c8 Chlorhexidine) on a Phadia UniCAP ${ }^{\text {тм }} 100$
Analyzer. The ImmunoCAP assay was performed following the manufacturer's specification in the protein reference unit of the hospital (measurement range: $<0.10$ to $>100 \mathrm{kU}_{\mathrm{A}} / \mathrm{L}$ ).

In vitro assay for chlorhexidine-specific IgE was used in preference to in vivo SPT to detect sensitization to chlorhexidine for the following reasons:

- The IgE immunoassay is a validated standardized blood test, carries an increased acceptance rate among participants, and is more objective than the more timeconsuming SPT.

- SPT requires standardized chlorhexidine reagents, technical expertise, and experience to interpret results reliably and carries a risk of interobserver variation.

- In allergy diagnosis, a good correlation exists between allergen-specific IgE levels and carefully conducted SPTs [41-43].

Case identification: Based on the previous studies [42] and following the manufacturer's suggestion, samples with chlorhexidine-specific IgE levels of $>0.35 \mathrm{kU}_{\mathrm{A}} / \mathrm{L}$ were considered positive.

Estimation of occupational exposure to chlorhexidine: Chlorhexidine $(2 \%-4 \%)$ is used in all hospital clinical areas, but the degree of exposure depends on healthcare work experience and the frequency of disinfectant use. The questionnaire asked the participants about these parameters to determine their occupational exposure: no exposure, low exposure, or high exposure.

\section{Data Analysis}

The blood test results and questionnaire responses were entered into Microsoft Excel spreadsheets for data analysis. Point estimates and 95\% CIs for the prevalence of seropositivity for chlorhexidine-specific IgE antibodies were calculated. In the statistical analysis, SPSS was used.

Logistic regression analysis was used to determine the relationships between a binary outcome of interest (e.g., seropositivity or seronegativity for chlorhexidine-specific IgE) and multiple risk factors. Logistic regression is useful for describing the relationship between independent variables (e.g., age and sex) and a binary or dichotomous response variable, expressed as a probability with only two possible values, such as positive or negative. A logistic model also allows selecting a subset of risk factors and best describes the relationship between these risk factors and the probability of an individual being seropositive for chlorhexidine-IgE, given their personal characteristics.

Based on the Pearson chi-square test results, Cramér's $V$ was also used for the statistical analysis to calculate the correlation and determine the association strengths after 


\section{Nursing \& Healthcare International Journal}

the significance was determined using the chi-square test. Cramér's V ranges from 0 (zero) to 1 ; when close to 0 , it shows little association between variables, and when close to 1 , it indicates a strong association.

\section{Results}

The results are summarized in Table 1.

\begin{tabular}{|c|c|c|c|c|}
\hline & \multicolumn{3}{|c|}{ Exposure } & \multirow{4}{*}{ All } \\
\hline Healthcare work experience & \multirow{2}{*}{$\begin{array}{c}\text { Yes } \\
>10 \\
\end{array}$} & \multirow{2}{*}{$\begin{array}{c}\text { Yes } \\
<10 \\
\end{array}$} & \multirow{2}{*}{$\begin{array}{c}\text { No } \\
\text { None } \\
\end{array}$} & \\
\hline Frequency of disinfectant use (per day) & & & & \\
\hline Occupational exposure & High & Low & None & \\
\hline Numbers (\%) & $99(42.5 \%)$ & $42(18.0 \%)$ & $92(39.5 \%)$ & $233(100 \%)$ \\
\hline Prevalence of chlorhexidine-specific IgE & $3(3.0 \%)$ & $1(2.4 \%)$ & $0(0 \%)$ & $4(1.72 \%)$ \\
\hline \multicolumn{5}{|c|}{ Work-related allergic symptoms } \\
\hline Skin symptoms & $40(40.4 \%)$ & $13(30.1 \%)$ & $0(0 \%)$ & $53(22.3 \%)$ \\
\hline Mucosal symptoms (eyes and nasal) & $11(11.1 \%)$ & $19(45.2 \%)$ & $0(0 \%)$ & $30(12.9 \%)$ \\
\hline Respiratory symptoms & $2(2.0 \%)$ & $3(7.1 \%)$ & $0(0 \%)$ & $5(2.1 \%)$ \\
\hline \multicolumn{5}{|c|}{ Atopic conditions } \\
\hline Hay fever & $25(25.2 \%)$ & $14(33.3 \%)$ & $16(17.4 \%)$ & $55(23.6 \%)$ \\
\hline Asthma & $9(9.1 \%)$ & $6(14.3 \%)$ & $1(1.1 \%)$ & $16(6.9 \%)$ \\
\hline Eczema & $9(9.1 \%)$ & $6(14.3 \%)$ & $1(1.1 \%)$ & $16(6.9 \%)$ \\
\hline Other allergies (food, drugs, and animals) & $44(44.4 \%)$ & $16(38.1 \%)$ & $4(4.3 \%)$ & $60(25.7 \%)$ \\
\hline On antiallergic treatment & $8(8.1 \%)$ & $2(4.8 \%)$ & $0(0 \%)$ & $10(4.3 \%)$ \\
\hline Nonwork-related allergic symptoms & $25(25.2 \%)$ & $15(35.7 \%)$ & $0(0 \%)$ & $40(17.2 \%)$ \\
\hline
\end{tabular}

Table 1: Summary of results $(n=233)$.

\section{Response Rate}

With a target sample size of 250,300 healthcare workers were invited to participate; 51 healthcare workers did not respond or declined to participate, 13 invitations were given to staff members who did not satisfy the inclusion criteria, and three healthcare workers withdrew their consent. The remaining respondents (233) completed the questionnaire and allowed blood sampling to analyze chlorhexidine- specific IgE antibodies. Therefore, the response rate in the study was $77.7 \%$.

\section{Age and Sex Distributions}

As shown in Table 2, 148 participants were female (63.5\%), and 85 were male (36.5\%). The mean age of the participants was 29.12 years (range, 18-62 years).

\begin{tabular}{|c|c|c|}
\hline & Means & Standard deviations \\
\hline Age & 29.12 years & 11.18 \\
\hline Healthcare experience & 62.94 months & 54.38 \\
\hline
\end{tabular}

Table 2: Means and standard deviations for age and work experience.

\section{Occupational Exposure}

Ninety-nine participants $(42.5 \%)$ were categorized as having high exposure to chlorhexidine (based upon their work experience and the frequency of disinfectant use), and $42(18 \%)$ had low exposure. Ninety-two participants $(39.5 \%)$ had minimal or no healthcare work experience. The mean duration of occupational exposure to chlorhexidine was 62.94 months (median, 48 months; range, 12-372 months).

\section{Prevalence of IgE-Mediated Chlorhexidine Hypersensitivity}

Four healthcare workers tested positive for chlorhexidine-specific antibodies, with an overall prevalence 
of $1.72 \%$ (95\% CI, $0.05 \%-3.39 \%$ ) in the study population. The prevalence increased to $2.82 \%(4 / 141)$ in the exposure groups. Work-related skin symptoms were more common in the high-exposure group, but mucosal and respiratory symptoms were more common in the low-exposure group.
Atopic conditions (e.g., hay fever, asthma, and eczema) and nonwork-related allergic symptoms appeared more prevalent in the exposed group (high and low) (Figures 1 \& 2).

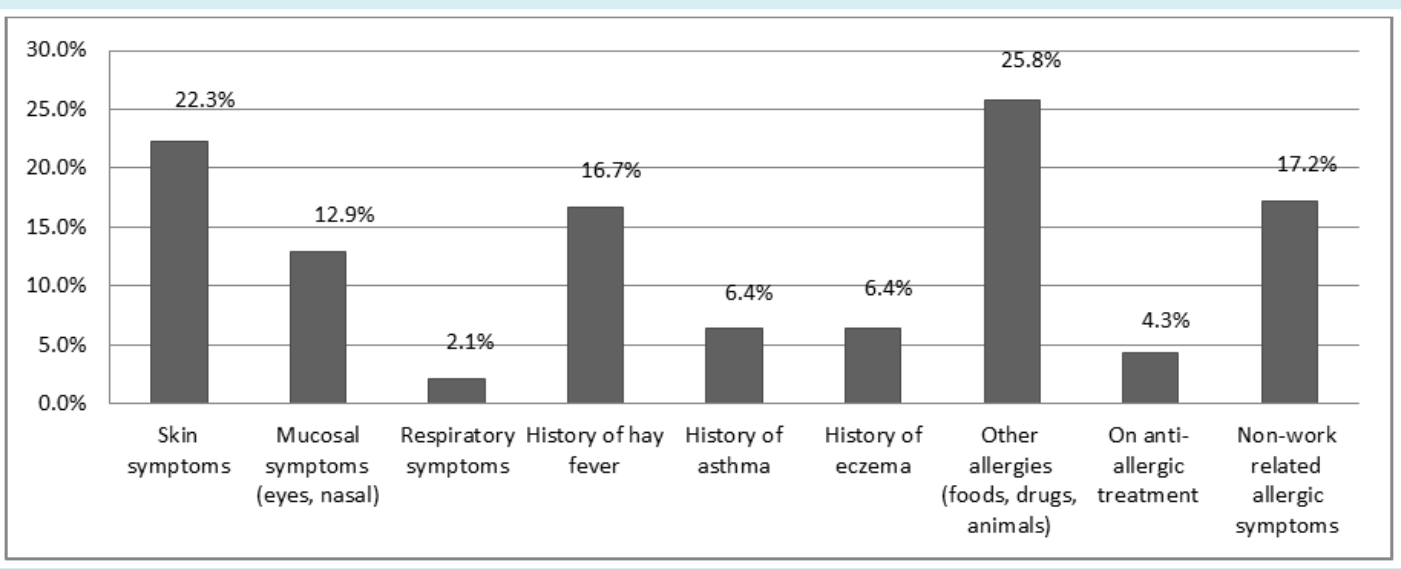

Figure 1: Shows the proportion of all the participants who reported allergic symptoms and risk factors.

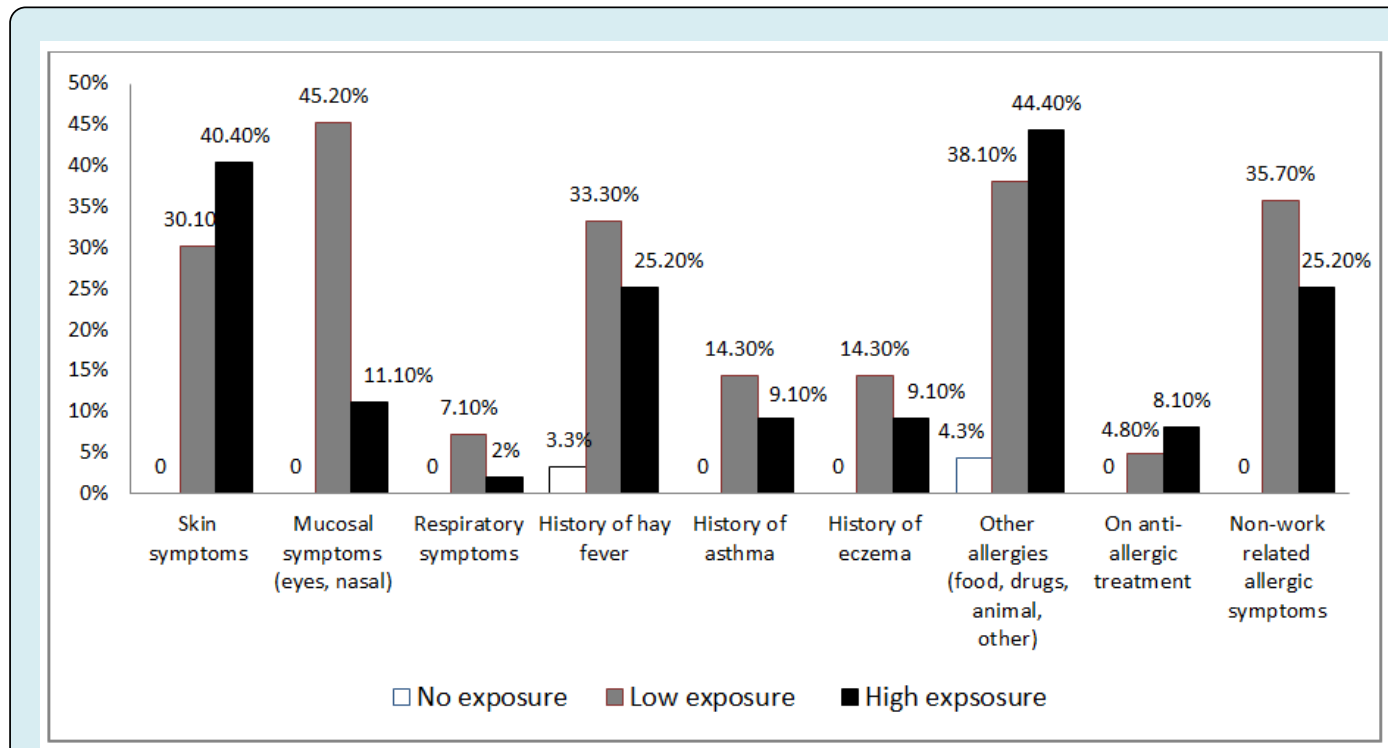

Figure 2: Shows the same attributes in different exposure groups.

\section{Statistical Analysis}

Regression and chi-square $\left(\chi^{2}\right)$ tests were used to analyze the data to provide a clearer picture of the influences of the variables and examine the data from many angles. As the duration of occupational exposure (healthcare experience) is the only independent continuous variable, the results would be best understood by analyzing the rest of the dichotomous data using nonparametric tests. However, reliable calculations were not possible because of the small number of positive cases.

\section{Predictors of Positive Chlorhexidine Ige: Binary Logistic Regression}

The dependent variable was the immunoassay result for chlorhexidine-specific IgE. As this was discrete, logistic regression was used to estimate the factors or variables that 
could influence IgE blood results. Using SPSS (version 16.0), variables such as various allergies, atopic conditions, and nonwork-related allergic symptoms were poor predictors of an IgE-positive blood test result. The following results indicate that adding the predictor variables to the model did not significantly increase the ability to detect whether a healthcare worker will test positive for IgE:

$\chi^{2}=10.46, p=3.14$, degree of freedom $=9, n=233, r^{2}=0.04$

The low $r^{2}$ (Cox and Snell) indicates a weak relationship among the variables.

\section{Predictors of Positive Chlorhexidine IgE: Chi- Square Analysis}

A two-way contingency table analysis was performed to evaluate whether healthcare workers' IgE immunoassay results were affected by atopic conditions and allergies. The variables were blood test results (for chlorhexidinespecific IgE) and hay fever; asthma; eczema; allergies to food, latex, metal, animal, or drugs; and handwashing frequency, all analyzed separately. Only two significant associations were found, that is, between drug allergies and duration of occupational exposure, as summarized in Table 3.

\begin{tabular}{|c|c|c|c|c|}
\hline Comparison & Pearson chi-square $\boldsymbol{\chi}^{\mathbf{2}} \mathbf{)}$ & $\boldsymbol{p}$ Value & $\boldsymbol{d f}$ & Cramér's $\boldsymbol{V}$ \\
\hline IgE +ve vs. drug allergy & 5 & $<0.05$ & 1 & 0.14 \\
\hline $\begin{array}{c}\text { IgE +ve } \boldsymbol{v s} \text {. duration of } \\
\text { exposure }\end{array}$ & 6.32 & $<0.05$ & 2 & 0.16 \\
\hline $\begin{array}{c}\text { (n = 233, } d f=\text { degree } \\
\text { of freedom, IgE +ve } \\
=\text { positive cases for } \\
\text { chlorhexidine-specific } \\
\text { IgE) }\end{array}$ & & & & \\
\hline
\end{tabular}

Table 3: Association between IgE-positive cases, drug allergy, and duration of exposure.

The associations between the IgE-positive cases and various exposure groups were evaluated through follow- up pairwise comparisons. Table 4 shows the results of the analysis.

\begin{tabular}{|c|c|c|c|}
\hline Comparison & Pearson chi-square $\boldsymbol{\chi}^{2} \mathbf{)}$ & $\boldsymbol{p}$ Value & Cramér's $\boldsymbol{V}$ \\
\hline No exposure $\boldsymbol{v s}$. low exposure & 3.8 & 0.05 & 0.14 \\
\hline No exposure vs. high exposure & 6.79 & 0.01 & 0.18 \\
\hline Low exposure vs. high exposure & 0.26 & 0.6 & 0.05 \\
\hline
\end{tabular}

Table 4: Pairwise comparisons of the IgE-positive cases and exposure groups.

The probability of someone having a negative blood test result was 395 times higher in the nonexposed group than in the low-exposure group and 241 times higher than in the high-exposure group. No significant differences were found between the high- and low-exposure groups. By using the chi-square analysis, skin symptoms and occupational exposure were also found to be significantly related (Pearson $\chi^{2}=28.14, \mathrm{p}<0.05, d f=2, \mathrm{n}=233$; Cramér's $V=0.34$ ). Followup pairwise comparisons were performed to evaluate the differences among these proportions. Table 5 shows the results of the analysis.

\begin{tabular}{|c|c|c|c|}
\hline Comparison & $\begin{array}{c}\text { Pearson chi-square } \\
\left(\boldsymbol{\chi}^{2} \mathbf{)}\right.\end{array}$ & $\boldsymbol{p}$ Value & Cramér's $\boldsymbol{V}$ \\
\hline No exposure vs. low exposure & 7.22 & 0.01 & 0.2 \\
\hline No exposure vs. high exposure & 27.93 & 0 & 0.37 \\
\hline Low exposure vs. high exposure & 2.31 & 0.12 & 0.15 \\
\hline
\end{tabular}

Table 5: Results of the pairwise comparisons of skin symptoms and exposure.

The probability of having a skin symptom was 163 times lower in the nonexposed group than in the low-exposure group and 63 times lower than in the high-exposure group. No significant differences in skin symptoms were found 
between the high- and low-exposure groups. All other comparisons (sex, atopic conditions, latex allergy, food allergy, and animal allergy) were not significant at $p>0.05$.

\section{Positive Cases}

All individuals who tested positive for chlorhexidinespecific IgE belonged to the exposed groups, and the mean length of their occupational exposures was 33 months (range: 24-48 months). Three of four positive cases used disinfectant $>10$ times per day (Table 6 ). None of the positive cases had type I hypersensitivity symptoms (urticaria, rhinoconjunctivitis, and angioedema), but three had other nonspecific skin symptoms such as redness, dryness, and itching. Three participants indicated type I hypersensitivity symptoms after exposure to chlorhexidine at work, but their immunoassay results for chlorhexidine-specific IgE were negative.

\begin{tabular}{|c|c|c|c|c|}
\hline & Case 1 & Case 2 & Case 3 & Case 4 \\
\hline Chlorhexidine-specific IgE (kUA/L) & 2.06 & 1.64 & 2.36 & 0.6 \\
\hline Sex, age & F, 24 & M,40 & F, 20 & M, \\
\hline Occupation & Senior medical student & Senior nurse & Paramedic & Allied healthcare worker \\
\hline Chlorhexidine exposure & Yes & Yes & Yes & Yes \\
\hline Disinfectant use (times/day) & $11-15$ & $11-15$ & $11-15$ & $<5$ \\
\hline Healthcare experience (months) & 48 & 36 & 24 & 24 \\
\hline Urticaria & No & No & No & No \\
\hline Rhinoconjunctivitis & No & No & No & No \\
\hline Angioedema & No & No & No & No \\
\hline Anaphylaxis & No & No & No & Yes \\
\hline Redness, dryness, and itching & No & Yes & Yes & Yes \\
\hline Handwashing & - & Yes & Not known & No \\
\hline Atopic conditions & None & Yes & Yes & None \\
\hline Other allergies & None & Yes & Yes & None \\
\hline On antiallergic treatment & None & Yes & None & None \\
\hline
\end{tabular}

Table 6: Positive cases of IgE-mediated chlorhexidine sensitivity.

\section{Discussion}

The study showed four cases with elevated levels of chlorhexidine-specific IgE, corresponding to an overall prevalence of $1.72 \%$ (2.82\% in the occupationally exposed group, 4/141 cases). In addition to four positive cases, three participants reported type I hypersensitivity symptoms (e.g., immediate urticaria and rhinoconjunctivitis) on exposure to chlorhexidine at work, but their blood test results were negative for chlorhexidine-specific IgE. It could be because of their hypersensitivity to other ingredients of chlorhexidinecontaining products.

A borderline result might have indicated avoidance behavior of the healthcare worker because of previous sensitization symptoms to chlorhexidine, which would have reduced IgE levels (from potentially positive to borderline). However, in the present study, all results $\left(<0.35 \mathrm{kU}_{\mathrm{A}} / \mathrm{L}\right)$ were reported as negative; therefore, borderline results (0.20-0.35 $\mathrm{kU}_{\mathrm{A}} / \mathrm{L}$ ) could not be identified; otherwise, the prevalence could potentially be much higher.

Chlorhexidine has been reported to cause acute hypersensitivity reactions when applied to intact skin [44], but this is a relatively uncommon phenomenon. Healthcare workers who wash their hands more frequently have drier and chapped skin, making them more vulnerable to sensitization by chlorhexidine. This would explain the comparatively higher prevalence of IgE-mediated chlorhexidine sensitivity in the high-exposure group.

The prevalence of IgE-mediated chlorhexidine hypersensitivity in this study should also be viewed in the context of two previous similar studies by Garvey, et al. (Denmark) and Nagendran, et al. (UK). In the Danish study, none of 104 healthcare workers was positive for 
chlorhexidine sensitization with the SPT and patch test [34]. In the UK, 4 (28\%) of 14 symptomatic healthcare workers had IgE-mediated chlorhexidine allergy [39]. The results of this study may not be directly comparable with the studies by Garvey, et al. and Nagendran, et al. as they were conducted in different settings, different concentrations, and sample sizes. However, like in the other two studies, it highlights the importance of the allergenic potential of chlorhexidine in occupational settings.

One aspect of the present study that differentiates it from previous studies is that it attempted to evaluate the possible association of chlorhexidine-specific IgE with multiple risk factors. The information obtained from the questionnaire on multiple demographics and occupational and clinical variables could help establish a relationship between several potential risk factors and the presence of chlorhexidinespecific IgE expression and clinical symptoms. However, because of the small number of positive cases, an accurate and reliable assessment of any association with multiple risk factors could not be achieved. The factor that appears most associated with chlorhexidine-specific IgE is the healthcare workers' cumulative exposure to chlorhexidine, which, in turn, depends on the frequency of handwashing and duration of healthcare work experience.

A weakness of this study is the difficulty of estimating occupational exposure to chlorhexidine accurately. The exposure estimates are subject to recall bias, and many participants described their work experience in nonspecific terms (e.g., >2 and >10 years).

Three of the four positive cases (Table 6) in this study had high occupational exposure to chlorhexidine for $>24$ months. Still, none of the four workers reported symptoms of IgE-mediated chlorhexidine hypersensitivity (rhinoconjunctivitis, urticaria, angioedema, asthma, and anaphylaxis). However, three patients reported nonspecific skin symptoms of pruritis, irritation, erythema, and fissuring, indicating that their skin symptoms could have been caused by type IV hypersensitivity (allergic contact dermatitis). This combined hypersensitivity (types I and IV) has been reported previously in the literature [24,45-48]. The significance of the combined hypersensitivity is unknown. Further work is required to establish whether the two types occur simultaneously and, if not, which one appears first.

Due to the low percentage of positive cases, it was difficult to demonstrate a statistical correlation between chlorhexidine-specific IgE antibodies and risk factors. The correlation between the IgE results and the degree of exposure, with a dose-response relationship, has been shown in Table 3. Statistically, however, the degree of exposure (low vs. high exposure) was not significantly associated with a positive result. In addition, reported skin symptoms (including type IV) also showed a statistical correlation with exposure ( $p=0.001)$. Again, exposure (low vs. high) was not relevant to whether individuals experienced skin symptoms, but whether exposure occurred was more important (Table 5). Sensitization to chlorhexidine may also happen through non-occupational exposure as it is an active ingredient in many over-the-counter preparations.

Nevertheless, compared with healthcare products, chlorhexidine is present in domestic products at a very low concentration (e.g., Savlon contains $0.1 \% \mathrm{w} / \mathrm{w}$ chlorhexidine), which may not be enough to cause sensitivity via intact skin or mucous membranes. Approximately $17 \%$ of the participants mentioned having allergic symptoms in the non-occupational context, but it was not possible to quantify their exposure.

Conversely, 92 participants did not have any occupational exposure to chlorhexidine and nonwork-related allergic symptoms, and no positive cases were found in the group. Therefore, it is unlikely that the study participants acquired chlorhexidine sensitivity through non-occupational exposure. In a study in Malaysia [49], 8.6\% of the participants without any known exposure to chlorhexidine showed sensitization, which suggests the potential future risk of developing hypersensitivity symptoms.

Potential risk factors such as the frequency and duration of chlorhexidine exposure and history of skin symptoms could also be subject to a recall bias. Finally, a "healthy worker effect" is possible in relation to the results of this study. Healthcare workers who experienced significant allergic symptoms to chlorhexidine or had multiple allergies may have changed their job roles or occupations to those involving very low or no exposure to chlorhexidine or disinfectants. Other healthcare workers might have switched to using alternative products after experiencing an initial adverse reaction to chlorhexidine. Such behavioral change would confound any relationship between past exposure to chlorhexidine and the presence of chlorhexidine-specific $\operatorname{IgE}$ antibodies at the time of their blood test for this study.

The current guidelines for hand hygiene also recommend $2 \%$ chlorhexidine, which appears to be the minimum bactericidal concentration, with $70 \%$ alcohol [11]. Evidence shows that chlorhexidine acts as a bacteriostatic agent at lower concentrations than a bactericidal agent $[7,8,10]$ and is less effective against certain organisms. Further work is needed in this area, and disinfection guidelines/protocols should consider effective disinfection and the potential risk to healthcare workers exposed to chlorhexidine. 
With its relatively larger sample size and evaluation of various risk factors, the present study could be considered a preliminary study. Further studies using larger or highrisk populations of healthcare workers and a more robust methodology may be conducted to confirm or dispute these findings.

\section{Conclusion}

The study showed that only $4(1.72 \%)$ of 233 participants had elevated IgE levels for chlorhexidine, suggesting that it is relatively safe in occupational settings. The study did not reveal a statistical correlation between the positive results and the degree of exposure (frequency of disinfectant use).

Despite the large sample size and the high response rate of this study, it was difficult to draw reliable conclusions because of the small number of positive cases, and a higher prevalence of IgE-mediated allergy among healthcare workers cannot be completely ruled out. In addition, because of the reported cases of combined type I and IV hypersensitivity reactions to chlorhexidine in the same individual, healthcare workers presenting with contact dermatitis (type IV hypersensitivity) should also be evaluated for type I hypersensitivity by measuring chlorhexidine-specific IgE levels.

Any changes in disinfectants and skin antiseptic protocols may not be recommended on the basis of the results of this study. With the ever-increasing use of chlorhexidine in combating healthcare-associated infections, including COVID-19, healthcare workers must be informed that the disinfectant can cause hypersensitivity reactions at the workplace. Chlorhexidine-sensitized individuals must be advised to avoid further exposure and provided with alternative handwashing agents.

Further studies in highly exposed healthcare workers (e.g., surgeons, theater practitioners, and renal and oncology staff) are recommended.

\section{Declarations}

\section{Ethics Approval and Consent to Participate}

Informed and voluntary consent was sought from all potential participants, who were allowed to withdraw their consent at any stage of the study. The St George's Hospital Research Ethics Committee approved the study. The project was sponsored by the Joint Research Office of St George's University, London, United Kingdom.

\section{Availability of Data and Materials}

The data and relevant material is available.

\section{Potential Competing Interests}

This work was supported by Phadia Limited (now Thermo Fisher Scientific), which provided chemical reagents for chlorhexidine-specific IgE tests, and they agreed to supply the reagent for 250 blood samples. However, Phadia Limited or any of its employees were not involved at any stage in the study design, data collection, analysis, interpretation, writing of the report, or the decision to submit this manuscript.

\section{Funding}

Not applicable

\section{Acknowledgments}

The author acknowledges the advice, guidance, and support from his supervisor, Dr. Samuel Thayalan, Consultant Occupational Physician at St. George's Hospital, London, UK. In addition, Dr. Vasantha Nagendran, Consultant Immunologist, National Health Services, UK, also provided useful guidance and professional advice during the project.

\section{References}

1. Davies, GE, Francis J, Martin AR, Rose FL, Swain G (1954) 1:6-Di-4'-chlorophenyldiguanidohexane (Hibitane). Laboratory investigation of a new antibacterial agent of high potency. Br JPharmacol 9(2): 192-196.

2. Larson E (1988) A causal link between handwashing and risk of infection? Examination of the evidence. Infect Control Hosp Epidemiol 9(1): 28-36.

3. Russell AD (1986) Chlorhexidine: antibacterial action and bacterial resistance. Infection 14(5): 212-215.

4. Mohammadi Z, Abbott PV (2009) The properties and applications of chlorhexidine in endodontics. Int Endod J 42(4): 288-302.

5. McDonnell G, Russell AD (2020) Antiseptics and disinfectants: activity, action, resistance. Clin Microbiol Rev 12(1): 147-179.

6. Richards RME, Cavill RH (1979) Electron-microscope study of the effect of chlorhexidine on Pseudomonas aeruginosa. Microbios 26(104): 85-93.

7. Dance DA, Pearson AD, Seal DV, Lowes JA (1987) A hospital outbreak caused by chlorhexidine- and antibiotic-resistant Proteus mirabilis. J Hosp Infect 10(1): 10-16.

8. Hammond SA, Morgan JR, Russell AD (1987) Comparative susceptibility of hospital isolates of gram-negative 
bacteria to antiseptics and disinfectants. J Hosp Infect 9(3): 255-264.

9. Shahid NS, Greenough WB, Samadi AR, Huq MI, Rahman N (1996) Hand washing with soap reduces diarrhoea and spread of bacterial pathogens in a Bangladesh village. J Diarrhoeal Dis Res 14(2): 85-89.

10. Russell AD, Day MJ (1993) Antibacterial activity of chlorhexidine. J Hosp Infect 25(4): 229-238.

11. Pratt RJ, Pellowe CM, Wilson JA, Loveday HP, Harper PJ, et al. (2007) National evidence-based guidelines for preventing healthcare-associated infections in NHS hospitals in England. J Hosp Infect 65S: S1-64.

12. Huang JT, Huang JT (2021) Use of chlorhexidine to eradicate oropharyngeal SARS-CoV-2 in COVID-19 patients. J Med Virol 93(7): 4370-4373.

13. Calnan CD (1962) Contact dermatitis from drugs. Proc $\mathrm{R}$ Soc Med 55(1): 39-42.

14. Reynolds NJ, Harman RM (1990) Allergic contact dermatitis from chlorhexidine diacetate in a skin swab. Contact Dermatitis 22(2): 103-128.

15. Osmundsen PE (1982) Contact dermatitis to chlorhexidine. Contact Dermatitis 8(2): 81-83.

16. Lasthein Andersen B, Brandrup F (1985) Contact dermatitis from chlorhexidine. Contact Dermatitis 13(5): 307-309.

17. Ljunggren $B$, Moller $H$ (1972) Eczematous contact allergy to chlorhexidine. Acta Dermato-venereologica; 52(4): 308-310.

18. Neering $H$, van Ketel WG (1972) Contact allergie door chlorhexidine. Nederlands Tijdschriftvoor Geneeskunde 116: 1742-1743.

19. Roberts DL Summerly R, Byrne JPH (1981) Contact dermatitis due to the constituents of Hibiscrub. Contact Dermatitis 7(6): 326-328.

20. Wong WK, Goh CL, Chan KW (1990) Contact urticaria from chlorhexidine. Contact Dermatitis 22: 52.

21. Wahlberg JE, Wennersten G (1971) Hypersensitivity and photosensitivity to chlorhexidine. Dermatologica 143(6): 376-379.

22. Ebo DG, Stevens WJ, Bridts CH, Matthieu L (1998) Contact allergic dermatitis and life-threatening anaphylaxis to chlorhexidine. J Allergy Clin Immunol 101: 128-129.

23. Sharp G, Green S, Rose M (2016) Chlorhexidine- induced anaphylaxis in surgical patients: a review of the literature. ANZ J Surg 86(4): 237-243.

24. Odedra KM, Farooque S (2014) Chlorhexidine: an unrecognized cause of anaphylaxis. Postgrad Med J 90(1070): 709-714.

25. Cogné Y, Mouton Faivre C, Cavasino T, Teychene AM, Pontual L, et al. (2017) Chlorhexidine-induced IgEmediated allergy in a 6-year-old child. J Allergy Clin Immunol Pract 5(3): 837-838.

26. Garvey LH, Roed Petersen J, Husum B (2001) Anaphylactic reactions in anaesthetised patients - four cases of chlorhexidine allergy. Acta Anaesthesiol Scand 45(10): 1290-1294.

27. Parkes AW, Harper N, Herwadkar A, Pumphrey R (2009) Anaphylaxis to the chlorhexidine component of Instillagel: a case series. Br J Anaesth 102(1): 65-68.

28. Stables GI, Turner WH, Prescott S, Wilkinson SM (1998) Generalized urticaria after skin cleansing and urethral instillation with chlorhexidine-containing products. Br J Urol 82: 756-757.

29. Stephens R, Mythen M, Kallis P, Davies DW, Egner W, et al. (2001) Two episodes of life-threatening anaphylaxis in the same patient to a chlorhexidine-sulphadiazinecoated central venous catheter. Br J Anaesth 87(2): 306308.

30. Pittaway A, Ford S (2002) Allergy to chlorhexidinecoated central venous catheters revisited. Br J Anaesth 88(2): 304-305.

31. Oda T, Hamasaki J, Kanda N, Mikami K (1997) Anaphylactic shock induced by an antiseptic-coated central venous catheter. Anesthesiology 87(5): 12421244.

32. Terazawa E, Shimonaka H, Nagase K, Masue T, Dohi $S$ (1998) Severe anaphylactic reaction due to a chlorhexidine-impregnated central venous catheter. Anesthesiology 89: 1296-1298.

33. Torricelli R, Wüthrich B (1996) Life-threatening anaphylactic shock due to skin application of chlorhexidine. Clin Exposure Allergy 26(1): 112.

34. Garvey LH, Roed Petersen J, Husum B (2003) Is there a risk of sensitization and allergy to chlorhexidine in health care workers? Acta Anaesthesiol Scand 47: 720724.

35. Grzybowski M, Ownby DR, Peyser PA, Johnson CC, Schork MA, et al. (1996) The prevalence of anti-latex 
IgE antibodies among registered nurses. J Allergy Clin Immunol 98(3): 535-544.

36. Waclawski ER, McAlpine LG, Thomson NC (1989) Occupational asthma in nurses caused by chlorhexidine and alcohol aerosols. BMJ 298(6678): 929-930.

37. Toletone A, Dini G, Massa E, Bragazzi NL, Pignatti P (2018) Chlorhexidine-induced anaphylaxis occurring in the workplace in a healthcare worker: case report and review of the literature. Med Lav 109(1): 68-76.

38. Sato K, Kusaka Y, Suganuma N, Nagasawa S, Deguchi Y (2004) Occupational allergy in medical doctors. J Occup Health 46(2): 165-170.

39. Nagendran V, Wicking J, Ekbote A, Onyekwe T, Garveyet LH (2009) IgE-mediated chlorhexidine allergy; a new occupational hazard? Occup Med 59(4): 270-292.

40. Daniel WW (1999) Biostatistics: a foundation for analysis in the health sciences, $7^{\text {th }}$ (Edn.), New York: John Wiley \& Sons.

41. Garvey LH (2006) Anaphylaxis to chlorhexidine. New Horizons Allergy 2: 1-5.

42. Oryszczyn MP, Annesi I, Neukirch F, Doré MF, Kauffmann F (1995) Longitudinal observation of serum IgE and skin prick test response. Am J Respir Crit Care Med 151(3): 663-668.
43. Ebo DG, Bridts CH, Stevens WJ (2006) IgE mediated anaphylaxis from chlorhexidine: diagnostic possibilities. Contact Dermatitis 55(5): 301-302.

44. Autegarden JE, Pecquet C, Huet S, Bayrou O, Leynadier F (1999) Anaphylactic shock after application of chlorhexidine to unbroken skin. Contact Dermatitis 40(4): 215.

45. Soderstrom L, Kober A, Ahsted, de Groot SH, Lange CE, et al. (2003) A further evaluation of the clinical use of specific IgE antibody testing in allergic diseases. Allergy 58(9): 921-928.

46. Lauerma AI (2001) Simultaneous immediate and delayed hypersensitivity to chlorhexidine digluconate. Contact Dermatitis 44(1): 59.

47. Thune $P$ (1998) Two patients with chlorhexidine allergy - anaphylactic reactions and eczema. Tidsskr Nor Laegeforen 118(21): 3295-3296.

48. Bergqvist Karlsson A (1988) Delayed and immediatetype hypersensitivity to chlorhexidine. Contact Dermatitis 18(2): 84-88.

49. Khazin SM, Abdullah D, Cheen Liew AK, Harun NA, Abdullah N, et al. (2019) IgE-mediated hypersensitivity to chlorhexidine among first-year dental students. Allergo J Int 28: 204-208. 